\title{
THE INCIDENCE OF STELLAR MERGERS AND MASS GAINERS AMONG MASSIVE STARS
}

\author{
S. E. DE MinK ${ }^{1,2,5}$, H. SANA ${ }^{3}$, N. LANGER ${ }^{4}$, R. G. IZZARD ${ }^{4}$, AND F. R. N. SCHNEIDER ${ }^{4}$ \\ ${ }^{1}$ Observatories of the Carnegie Institution for Science, 813 Santa Barbara St., Pasadena, CA 91101, USA \\ ${ }^{2}$ Cahill Center for Astrophysics, California Institute of Technology, Pasadena, CA 91125, USA \\ ${ }^{3}$ Space Telescope Science Institute, Baltimore, MD 21218, USA \\ ${ }^{4}$ Argelander Institut für Astronomie der Universität Bonn, Germany \\ Received 2013 October 4; accepted 2013 December 11; published 2014 January 20
}

\begin{abstract}
Because the majority of massive stars are born as members of close binary systems, populations of massive mainsequence stars contain stellar mergers and products of binary mass transfer. We simulate populations of massive stars accounting for all major binary evolution effects based on the most recent binary parameter statistics and extensively evaluate the effect of model uncertainties. Assuming constant star formation, we find that $8_{-4}^{+9} \%$ of a sample of early-type stars are the products of a merger resulting from a close binary system. In total we find that $30_{-15}^{+10} \%$ of massive main-sequence stars are the products of binary interaction. We show that the commonly adopted approach to minimize the effects of binaries on an observed sample by excluding systems detected as binaries through radial velocity campaigns can be counterproductive. Systems with significant radial velocity variations are mostly pre-interaction systems. Excluding them substantially enhances the relative incidence of mergers and binary products in the non-radial velocity variable sample. This poses a challenge for testing single stellar evolutionary models. It also raises the question of whether certain peculiar classes of stars, such as magnetic $\mathrm{O}$ stars, are the result of binary interaction and it emphasizes the need to further study the effect of binarity on the diagnostics that are used to derive the fundamental properties (star-formation history, initial mass function, mass-to-light ratio) of stellar populations nearby and at high redshift.
\end{abstract}

Key words: binaries: close - Galaxy: stellar content - stars: early-type - stars: massive

Online-only material: color figures

\section{INTRODUCTION}

Young massive stars are predominantly found in close binary systems (e.g., Mason et al. 2009; Sana \& Evans 2011; Chini et al. 2012). This implies that the majority of massive stars interact with a companion before ending their lives as supernovae (Sana et al. 2012). Such interaction has drastic consequences for the further evolution and final fate of both stars (e.g., Podsiadlowski et al. 1992; Wellstein \& Langer 1999; Eldridge et al. 2008) and gives rise to a variety of exotic phenomena including blue stragglers, X-ray binaries, millisecond pulsars, and gamma-ray bursts.

The strong preference for close systems, with orbital periods of less than a few days, implies that a third of the systems interact while both stars are still on the main sequence (Sana et al. 2012). Binary evolutionary models predict that a large fraction of these systems evolve into a contact configuration (e.g., Pols 1994; Wellstein et al. 2001; Nelson \& Eggleton 2001; de Mink et al. 2007). The evolution of massive contact binaries is uncertain, but it is anticipated that the stars are driven deeper into contact due to the continuing expansion of the stars and shrinkage of the orbit due to angular momentum loss. In particular, the loss of mass with high specific angular momentum through the outer Lagrangian point, possible torques resulting from circumbinary material, and dissipative processes occurring in the common envelope are believed to eventually result in a merger of the two stars (e.g., Podsiadlowski et al. 1992; Wellstein et al. 2001).

Recently, the merger of a close binary system, although at lower mass, was caught in the act as the transient V1309 Sco (Tylenda et al. 2011). It has also been suggested that the light

\footnotetext{
5 Einstein Fellow.
}

echo of V838 Mon resulted from a massive merger (Munari et al. 2002; Tylenda \& Soker 2006), but this case remains more controversial.

For massive stars, the fraction of stars that will merge as a result of contact in a close binary is predicted to be as high as $20 \%-30 \%$ according to the most recent binary statistics (Sana et al. 2012). In addition, mergers may be triggered by dynamical interactions between three-body systems (e.g., Kozai 1962; Perets \& Fabrycky 2009; Hamers et al. 2013) or as a result of (multiple) collisions in dense star clusters (Portegies Zwart et al. 2004; Glebbeek et al. 2009) or near the galactic center (Antonini et al. 2010, 2011).

The chance of witnessing the merger event of two massive stars is small because of the scarcity of massive stars. The rate of such events in our galaxy is expected to be about $20 \%-30 \%$ of the Galactic supernova rate, about one every 200 years (e.g., Langer 2012). In contrast, the products of such mergers may be rather common, in particular for mergers between two main sequence stars. These objects are expected to be rejuvenated as fresh gas is mixed into the central burning regions (e.g., Glebbeek et al. 2013). These merger products are expected to be among the brightest stars in young clusters forming a massive analogue of blue stragglers (e.g., Mermilliod 1982; Chen \& Han 2009; Lu et al. 2010; Schneider et al. 2014).

For the population of early-type stars in a typical galaxy, which is not characterized by a single burst of star formation, mergers and mass gainers cannot easily be identified. However, if such stars are abundant, they can in principle affect various diagnostics that are used to derive the fundamental properties such as the star-formation history and initial mass function (e.g., van Bever \& Vanbeveren 1998; Eldridge 2012; Zhang et al. 2012; Li et al. 2012). Such properties are generally derived 
Table 1

Impact of Uncertainties in the Initial Conditions and Physical Assumptions on the Incidence of Mergers and the Total Number of Binary Products Among a Population of Massive Early-type Stars

\begin{tabular}{|c|c|c|c|c|}
\hline & $\begin{array}{l}\text { Standard } \\
\text { Values }\end{array}$ & $\begin{array}{l}\text { Extreme } \\
\text { Values }\end{array}$ & $\begin{array}{c}\text { All Binary Products } \\
\text { (\%) }\end{array}$ & $\begin{array}{c}\text { Mergers Only } \\
(\%)\end{array}$ \\
\hline \multicolumn{5}{|c|}{ Initial conditions } \\
\hline Standard simulation for reference & & & 28.7 & 8.4 \\
\hline Primary mass distribution & $\alpha=2.35$ & $1.65,3.05$ & $26.8-32.1$ & $7.8-9.4$ \\
\hline Mass ratio distribution & $\kappa=0$ & $-1,1$ & $22.3-33.0$ & $9.9-7.1$ \\
\hline Orbital period distribution & $\pi=-0.5$ & $0,-1$ & $19.4-39.9$ & $\underline{3.5}-16.6$ \\
\hline Binary fraction & $f_{\text {bin }}=0.7$ & $0.3,1.0$ & $\underline{14.7-36.5}$ & $4.3-10.7$ \\
\hline Metallicity & $Z=0.2$ & $0.004,0.03$ & $\overline{26.5}-36.8$ & $4.5-9.0$ \\
\hline Brightness limit & $L_{\lim }\left(L_{\odot}\right)=10^{4}$ & $10^{5}$ & 34.2 & 12.2 \\
\hline \multicolumn{5}{|c|}{ Main physical assumptions } \\
\hline Standard simulation for reference & & & 27.3 & 8.0 \\
\hline Accretion efficiency & $\beta=\beta_{\mathrm{th}}$ & $\beta_{\mathrm{K}}, 1$ & $17.7-34.3$ & $7.4-7.2$ \\
\hline Angular momentum loss & $\gamma=\gamma_{\mathrm{acc}}$ & $0, \gamma_{\mathrm{L}}$ & $\overline{27.4}-\overline{24.5}$ & $8.1-8.4$ \\
\hline Contact during main sequence & $q_{\text {crit,MS }}=0.65$ & $0.25,0.8$ & $28.1-26.4$ & $\underline{6.6}-9.1$ \\
\hline Contact during Hertzsprung gap & $q_{\text {crit }, \mathrm{HG}}=0.4$ & $0, q_{W 01}$ & $21.7-23.0$ & $\overline{8.5}-8.4$ \\
\hline Treatment mergers: mass loss & $\mu_{\mathrm{loss}}=0.1$ & $0,0.25$ & $27.1-27.3$ & $7.8-8.0$ \\
\hline Treatment mergers: mixing & $\mu_{\operatorname{mix}}=0.1$ & 0,1 & $26.6-30.6$ & $7.1-12.3$ \\
\hline
\end{tabular}

Notes. Symbols are explained in Section 2. The underlined values are the largest deviation in each category.

a All binary products, i.e., mergers, systems undergoing Roche lobe overflow and companions after Roche lobe overflow.

using population synthesis models such as GALAXEV (Bruzual \& Charlot 2003), STARBURST99 (Leitherer et al. 2010), and FSPS (Conroy et al. 2009), in which all stars are in principle assumed to evolve in isolation. Estimating the incidence of binary products is therefore ultimately motivated by the need to improve our understanding of the validity of the properties derived for stellar populations nearby as well as those at high redshift.

A more direct motivation is the need to test state-of-theart stellar evolution models, which contain uncertain effects of convection, rotation, and magnetic fields (e.g., Brott et al. 2011a; Ekström et al. 2012; Potter et al. 2012). Lacking prescriptions from first principles makes calibration against observed populations indispensable. These models generally assume the stars to evolve in isolation. However, interaction with a binary companion can lead to drastic changes in the observable properties. To evaluate the validity of tests and calibrations of the models against observed samples, it is necessary to estimate the contamination of such samples with stars that are the products of binary interaction.

In this work we take a first step toward quantifying the implications of the newly derived binary fraction and distribution of orbital properties of massive binary stars (Sana et al. 2012). For this purpose we employ a rapid synthetic binary evolution code that has been updated to adequately describe the main relevant processes in Section 2. We simulate a population of young massive stars in a typical galaxy assuming continuous star formation to compute the incidence of mergers and other products of binary evolution. In particular, we examine which binary products can be detected as binary systems through radial velocity variations in Section 3.1. We assess the significance of our results by varying uncertain input distributions and the adopted treatment of uncertain physical processes in Section 3.2. In Section 4 we discuss the presence of binary products in observed samples and how they may be recognized and we conclude in Section 5.

\section{METHOD}

To estimate the effects of binary interaction on a population of early-type stars, we adopt the binary fraction and distribu- tion of orbital periods and mass ratios based on observations of O stars in nearby ( $\lesssim 3 \mathrm{kpc}$ ) young (about $2 \mathrm{Myr}$ ) star clusters, which have been subject to an intense spectroscopic monitoring campaign (Sana et al. 2012). Although the sample is of modest size, including 71 systems containing at least one $\mathrm{O}$ star, it exceeds any previous sample in completeness providing orbital solutions for over $85 \%$ of the detected binary systems. This allowed the derivation of the distribution of binary parameters corrected for incompleteness and biases, resulting in the following distribution of initial orbital periods, $p$,

$$
f_{p}(\log p) \sim(\log p)^{\pi}
$$

for $\log p$ (days) $\in[0.15,3.5]$, where $\pi=-0.55 \pm 0.2$ and a distribution of mass ratios $q$ defined as the mass of the less massive star over the mass of the more massive star,

$$
f_{q}(q) \sim q^{\kappa}
$$

for $q \in[0.1,1]$ where $\kappa=-0.1 \pm 0.6$. The binary fraction, i.e., the number of binary system with periods and mass ratios in the range specified with respect to the total number of single and binary systems, is $f_{\text {bin }}=0.69 \pm 0.09$. The distribution of primary masses is consistent with a mass function,

$$
f_{m}(m) \sim m^{-\alpha}
$$

with $\alpha=2.35$ (Salpeter 1955). Given the young ages of the clusters in this sample, we consider these in our simulations as initial conditions at the onset of hydrogen burning.

In our standard simulation we adopt $f_{\text {bin }}=0.7, \pi=-0.5$, $\kappa=0$, and $\alpha=2.35$. We consider variations on these parameters (see Table 1) that generously include the confidence interval quoted by Sana et al. (2012). The remaining fraction $1-f_{\text {bin }}$ is included as single stars, even though these may in reality have a nearby low mass companion or a companion in a wide orbit.

To model the effect of stellar evolution and binary interaction we employ a synthetic binary evolution code that is described in detail in de Mink et al. (2013, hereafter Paper I) and references therein. This code was originally developed by Hurley et al. (2000, 2002) and Izzard et al. (2004, 2006, 2009) based on 
stellar models by Pols et al. (1998). It has been updated and extended to include various processes relevant for this study (Paper I). A brief summary of the main aspects is given here.

We account for mass and angular momentum loss through winds (Nieuwenhuijzen \& de Jager 1990; Vink et al. 2001), effects of rotation on the stellar winds (Maeder \& Meynet 2000), and deformation due to rotation in the Roche approximation (Paper I). We adopt a metallicity of $Z=0.02$. We account for interaction through tides (Zahn 1977; Hurley et al. 2002) and Roche lobe overflow (Hurley et al. 2002) assuming circular orbits. In our standard simulation, we limit the accretion rate by the thermal rate of the accreting star (Tout et al. 1997; Hurley et al. 2002) and we assume that the remainder of the mass is lost from the system taking away the specific orbital angular momentum of the accreting star. Because the efficiency of mass transfer, $\beta$, defined as the fraction of the mass transferred from the donor star to the companion star that is actually accreted by the companion, and the specific angular momentum, $\gamma$, of material lost from the system are highly uncertain, we consider the extreme cases of conservative mass transfer $(\beta=1)$ and the highly non-conservative case where a star can no longer accrete after it has been spun up to its Keplerian limit (cf. Paper I). This latter case is indicated as $\beta=\beta_{\mathrm{K}}$ and is equal to the assumptions made in Petrovic et al. (2005) and de Mink et al. (2009). For the angular momentum loss we consider the extreme case that all mass is lost through the outer Lagrangian point (indicated as $\gamma=\gamma_{L}$ ) and the extreme limit where the specific angular momentum of the mass lost from the system is negligible $(\gamma=0)$.

We assume that binary systems come in contact when $M_{\text {acc }} / M_{\text {don }}<q_{\text {crit }}$ (e.g., Kippenhahn \& Meyer-Hofmeister 1977), where $M_{\text {acc }}$ and $M_{\text {don }}$ refer to the mass of the accreting star and the donor star. For systems with a main sequence donor star we adopt $q_{\text {crit,Ms }}=0.65$, based on a calibration against a grid of detailed binary evolutionary models (de Mink et al. 2007). Given the considerable uncertainties concerning the formation of contact (cf. Section 5.2.1 in Paper I) we explore the extreme cases of $q_{\text {crit,Ms }} \in\{0.25,0.75\}$. For systems with a Hertzsprung gap donor star we adopt $q_{\text {crit, HG }}=0.4$ and explore the effect of the extreme assumptions $q_{\text {crit, } \mathrm{HG}} \in\left\{0.0, q_{\mathrm{W} 01}\right\}$, where $q_{\mathrm{w} 01}=1.0$, for $p>30$ days and 0.65 for $p \leqslant 30$ days to mimic the detailed models by Wellstein et al. (2001). We follow Hurley et al. (2002) for the treatment of evolved donors.

We assume that contact binaries merge. During the coalescence we assume in our standard simulations that a fraction $\mu_{\text {loss }}=0.1$ of the system mass is lost during the merger taking away the excess angular momentum. When investigating the uncertainties we consider the case where mass loss can be neglected, $\mu_{\mathrm{loss}}=0$, and $\mu_{\mathrm{loss}}=0.25$. The merger product is assumed to settle to its thermal equilibrium structure while rotating near its Keplerian rotational velocity. It is assumed that the core of the most evolved star, which has the lowest entropy, sinks to the center of the merged star. In our standard simulation, only a small fraction $\mu_{\text {mix }}=0.1$ of the hydrogen-rich envelope is mixed into the new convective core (e.g., Gaburov et al. 2008; Glebbeek et al. 2013). We also consider the extreme case that the merger product is completely mixed $\mu_{\text {mix }}=1$, as is assumed in the original version of the code, and the case of no additional mixing $\mu_{\text {mix }}=0$. For accreting stars we account for rejuvenation by assuming that the star adapts it structure to its new mass, mixing in fresh hydrogen as the convective core expands (Paper I and references therein).

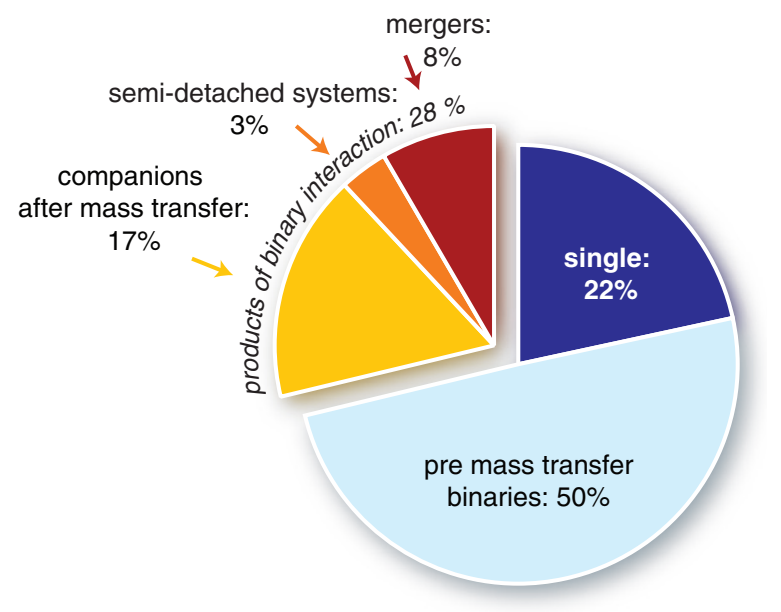

Figure 1. Incidence of stellar mergers and (post) mass transfer systems in our standard simulation of a population of massive main sequence stars assuming continuous star formation and an initial binary fraction of $70 \%$. Percentages are expressed in terms of the number of systems containing at least one main sequence star. See Section 3 for a discussion and Table 1 for the impact of model uncertainties on these predictions.

(A color version of this figure is available in the online journal.)

In our simulations of a population of early-type stars we select stars that are undergoing central hydrogen burning for which the combined brightness of the main-sequence components exceeds $10^{4} L_{\odot}$ (see Paper I). We chose this approach instead of estimating the spectral types directly from the effective temperatures because our predictions of the luminosity and evolutionary phase are more reliable than effective temperatures. The adopted limit corresponds in our models to the brightness of a single main-sequence star with initial mass $\gtrsim 8-12 M_{\odot}$, where the range reflects the fact that stars become brighter as they evolve over the main sequence. This roughly corresponds to stars of spectral types early B and $\mathrm{O}$. We consider continuous star formation, which is a good approximation for a large system with multiple bursts of star formation, such as the complete early-type star population in a typical galaxy. The case of different star formation histories (starbursts) computed with the same code and very similar assumptions are discussed in Schneider et al. (2014).

\section{THE INCIDENCE OF BINARY PRODUCTS IN A POPULATION OF MASSIVE EARLY-TYPE STARS}

The properties of our simulated stellar population are summarized in Figure 1, which indicates the relative contribution of single stars, binary stars, and products of binary interaction. The percentages are expressed in terms of the number of systems, either single or binary, that contain at least one main sequence star and for which the combined brightness of the main-sequence components exceeds $10^{4} L_{\odot}$. A binary system containing two main-sequence stars is counted only once.

The simulations started with $70 \%$ binaries and $30 \%$ single stars at birth. For the case of continuous star formation we find that the contribution of pre-interaction binaries is reduced from $70 \%$ at birth to $50 \%$ in the current population as a result of stellar evolution and binary interaction. About a fifth consist of stars that we refer to as single stars, even though some may have a companion in a very wide orbit or a very low mass companion, which are not accounted for in our simulation.

More than a quarter of the systems have been severely affected by interaction with their companion. This group mainly consists 

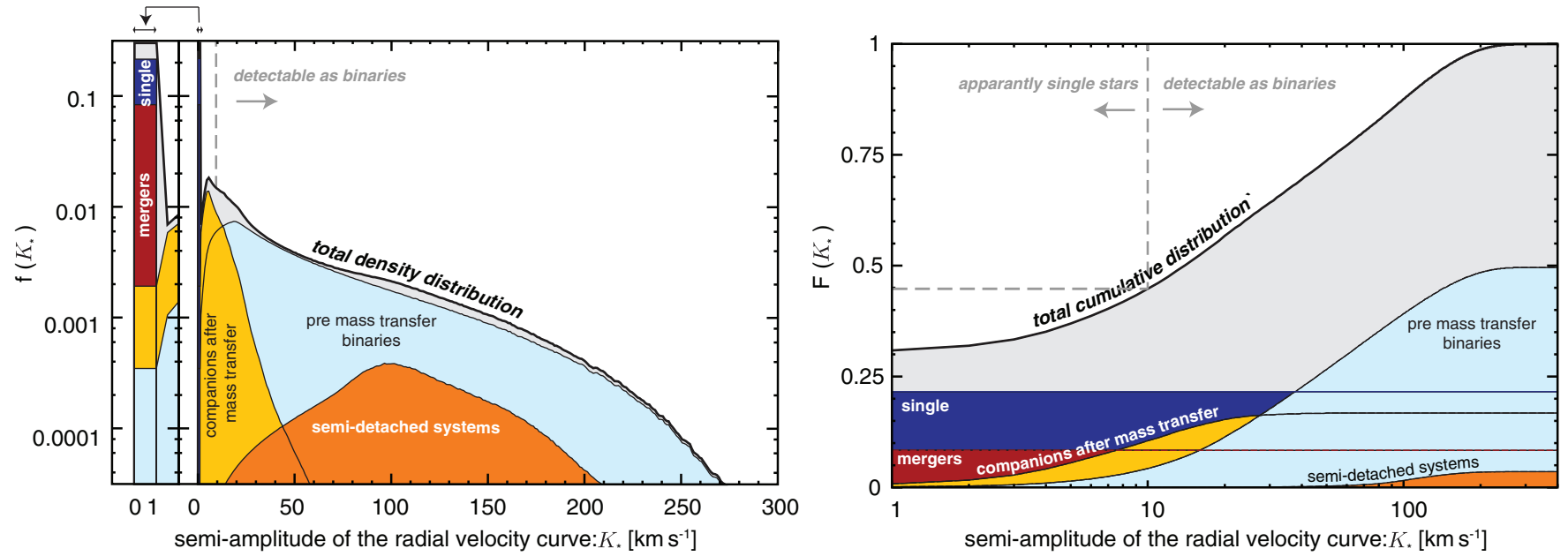

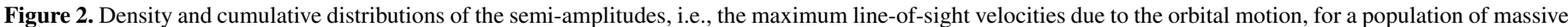

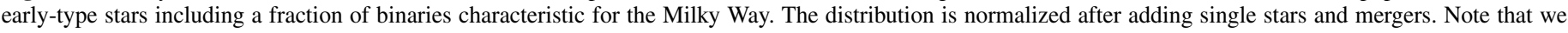
adopted logarithmic-linear and linear-logarithmic scales to enhance different features.

(A color version of this figure is available in the online journal.)

of mergers $(8 \%)$ and stars that have previously gained mass from a (former) companion (17\%). The fraction of systems that are in a semi-detached configuration, i.e., that are currently undergoing mass transfer through Roche lobe overflow, is small (3\%). This is because the mass transfer phase typically lasts for a few thermal timescales at most, which is short compared to the stellar lifetime. An exception are close systems that experience case A mass transfer (Nelson \& Eggleton 2001), i.e., mass transfer from a main-sequence donor, which can last for up to about a third of the main sequence lifetime (e.g., Figure 3 in de Mink et al. 2007). Practically all semi-detached systems, accounting for a few percent of the population, are undergoing slow Case A mass transfer. These systems form a subset of the observed 2 type systems.

The large fraction of mergers and mass gainers can be understood as the combined effect of different mechanisms. First, their production is favored by the large close binary fraction at birth. Second, binary products have gained mass through accretion or coalescence, resulting in an increase in brightness. Stars that were initially not massive and luminous enough to be included in our brightness limited sample can become bright enough after mass accretion. In other words, the binary products in our sample come from a wider range of initial masses than the single stars and stars that have not yet interacted. In addition, these binary products originate from lower mass systems, which are favored by the slope of the initial mass function. Third, when a main sequence star accretes mass it typically adapts its internal structure leading to an increase of the size of the convective core. As a result the hydrogen rich layers above the original core are mixed to the central burning regions providing fresh fuel, which effectively rejuvenates the star (Kippenhahn \& Meyer-Hofmeister 1977; Podsiadlowski et al. 1992; Braun \& Langer 1995; Dray \& Tout 2007; Claeys et al. 2011). The prolonged lifetime increases the fraction of stars that is expected to be observed after mass transfer.

\subsection{The Counterproductive Effect of Selecting against Binaries}

The contamination of a sample of early-type stars with binary products poses a challenge for their usefulness to test stellar models. A commonly adopted approach to try to reduce the effects of binaries on an observed sample is to exclude every object that is a known binary. Here, we demonstrate that removing detected binaries from a sample is counterproductive (cf. de Mink et al. 2011).

Spectroscopic binaries are detected through variations in the radial velocity resulting from the orbital motion. In a singlelined circular spectroscopic binary, the maximum radial velocity variation, $\Delta v$, that can be measured if the orbital phase is well sampled near quadrature, is equal to $2 K_{*}$, where $K_{*}$ is the semiamplitude of the radial velocity curve for the brightest star, and $i$ is the inclination angle. In a typical early-type system with a primary mass $M_{*}$, a mass ratio $q$, and an orbital period $p$,

$$
K_{*} \approx 80 \mathrm{~km} \mathrm{~s}^{-1}\left(\frac{\sin i}{\pi / 4}\right)\left(\frac{M_{*}}{20 M_{\odot}}\right)^{\alpha_{m}}\left(\frac{q}{0.5}\right)^{\alpha_{q}}\left(\frac{p}{10 \text { days }}\right)^{\alpha_{p}}
$$

where $\alpha_{m}=1 / 3$ and $\alpha_{p}=-1 / 3$ and the mass ratio dependence is approximated as a power law with exponent $\alpha_{q} \approx 0.86$ for $q \in[0.05,1]$.

Obtaining accurate radial velocity measurements for earlytype stars is challenging because there are relatively few suitable lines. Moreover, the lines are typically broad as a result of rotation and macro and micro turbulence. A typical accuracy that can be reached when measuring radial velocity variations is $1-10 \mathrm{~km} \mathrm{~s}^{-1}$ depending on the signal-to-noise ratio, the spectral type, and the rotation rate. Pulsations and stellar winds can further induce apparent variations of low amplitude. Therefore, radial velocity variations of $\Delta v \geqslant \Delta v_{\text {lim }} \equiv 20 \mathrm{~km} \mathrm{~s}^{-1}$ (or $K_{*} \geqslant 10 \mathrm{~km} \mathrm{~s}^{-1}$ ) is typically considered as an unambiguous sign of orbital motion due to the presence of a companion (e.g., Sana et al. 2013).

In Figure 2 we show the probability density function $f$ and the cumulative distribution $F$ of the semi-amplitudes of the binaries in our simulated population assuming random inclination angles. The distributions are normalized $f$ after adding single stars and mergers, for which we set $K_{*}=$ $0 \mathrm{~km} \mathrm{~s}^{-1}$. The detection limit is indicated by a vertical dashed line. As can been seen in the right panel, about $45 \%$ of the objects do not show any radial velocity variations that are large enough to be unambiguously detected as caused by a companion star. This group consists of stars that appear to be single.

The remaining 55\% show radial velocity variations larger than the detection limit. In principle these are detectable as 


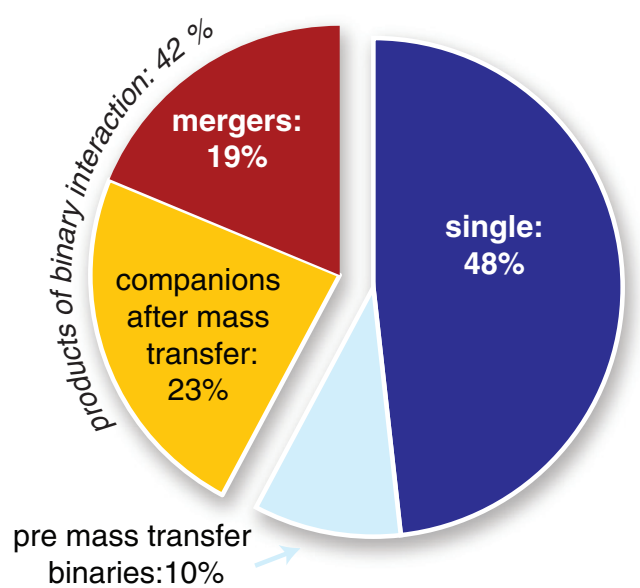

\section{(a) Apparently single $\left(K_{*}<10 \mathrm{~km} \mathrm{~s}^{-1}\right)$}

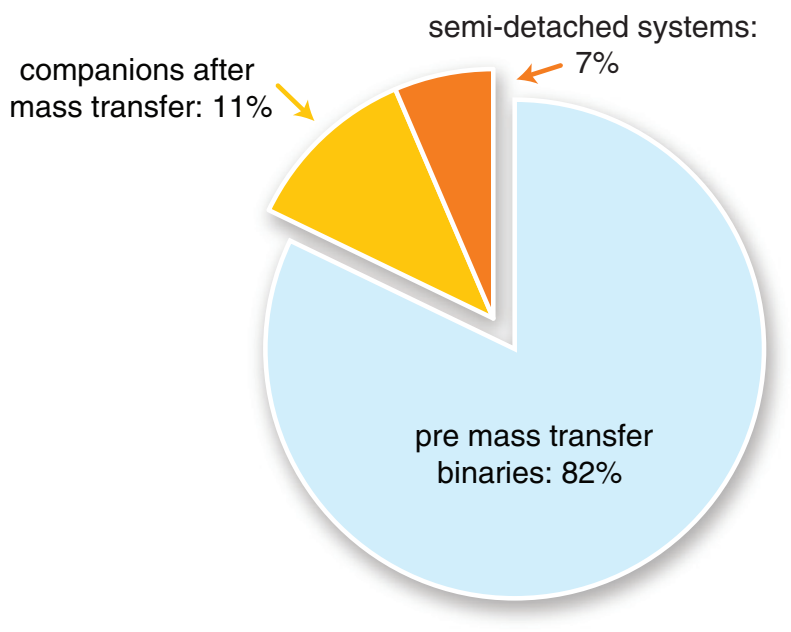

(b) Detectable as binary $\left(K_{\star}>10 \mathrm{~km} \mathrm{~s}^{-1}\right)$

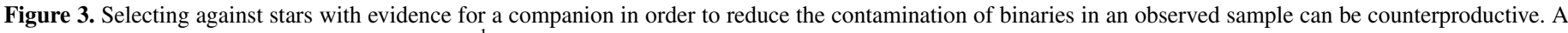

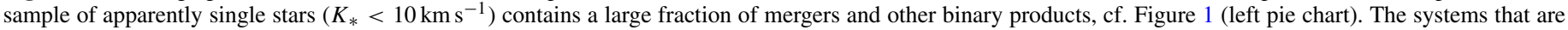
removed are preferentially binary systems that have not yet experienced interaction (right pie chart).

(A color version of this figure is available in the online journal.)

binaries. This requires multiple spectra to be taken that cover enough different phases of the orbit such that the full radial velocity curve can be reconstructed. For semi-amplitudes larger than the detection limit, the distribution is dominated by the primary stars of binary systems that have not yet interacted by mass transfer. Their semi-amplitudes extend beyond $250 \mathrm{~km} \mathrm{~s}^{-1}$, although semi-amplitudes of around $20 \mathrm{~km} \mathrm{~s}^{-1}$, approaching the detection limit, are most common. Systems that are currently undergoing Roche lobe overflow typically have semi-amplitudes around $100 \mathrm{~km} \mathrm{~s}^{-1}$, well above the detection limit. This group is dominated by mass transfer systems in which the Roche lobe filling star is a main-sequence star. These systems have compact orbits leading to large radial velocity variations.

The post interaction systems indicated as "companions after Roche lobe overflow" consist of a main-sequence star accompanied by a helium star, a neutron star, black hole, or white dwarf. These systems typically have small semi-amplitudes that are below the unambiguous detection limit. A significant fraction will be unbound as a result of the birth kick of the neutron star during the supernova explosion of the primary. Because the dependence of the birth kick distribution on the pre-explosion properties is highly uncertain, we assume a zero kick velocity in all cases, such that the quoted number of post-interaction systems with detectable radial velocity variations provides an upper limit.

When removing systems that are detected as radial velocity variables from a sample, one preferably removes binary systems from the sample that have not yet interacted. The products of binary interaction, which typically do not show measurable radial velocity variations, are left in the sample. Figure 3 summarizes the consequences for an idealized observing campaign, in which sufficient observing time is allocated to acquire full phase coverage of each system. In this case each binary with a semi-amplitudes $K_{*}>\frac{1}{2} \Delta v_{\text {lim }} \equiv 10 \mathrm{~km} \mathrm{~s}^{-1}$ would be identified.

The left pie chart shows the population of stars with no significant radial velocity variations, i.e., stars that appear to be single. About half of this sample are indeed stars that were born as single stars (48\%). A small fraction (10\%) corresponds to preinteraction binary systems displaying radial velocity variations that are too small to be detected. This group consists of the widest binaries and binaries with orbits that are aligned nearly face-on. These two groups together, accounting for $58 \%$ of the sample, are stars that have lived their lives so far without experiencing any significant interaction with a companion.

The remaining $42 \%$ consist of stars whose evolutionary history deviates strongly from that of an isolated star. These consist of mergers (19\%) and stars that have gained mass through Roche lobe overflow in the past $(27 \%)$. In reality systems with radial velocity variations approaching the detection limit are hard to detect without extensive monitoring. The pie chart on the right-hand side shows that those binaries that in principle can be detected through radial velocity variations. This sample consists preferentially of pre-interaction systems.

We conclude that excluding detected binaries from a sample to reduce the contamination of binary products is counterproductive. The binary products are typically not detected and will therefore represent a larger fraction of the sample than single stars. Those objects that are identifiable as binaries are dominated by stars that have not yet interacted. Apart from the closest systems in which tides play a role these systems have lived their lives effectively as single stars.

\subsection{Effect of Model Uncertainties}

In Table 1 we summarize the effect of varying the initial conditions and the main uncertain physical assumptions on the incidence of binary products and mergers in particular.

With regard to the adopted initial distributions of binary parameters, it is the power law exponent in the distribution of orbital periods that is the main cause for uncertainty. We find that the fraction of stars that are binary products varies from $19 \%$ to $40 \%$ when considering an initial distribution that 
is flat in $\log p$ (i.e., $\pi=0$ or "Opik's law") and a distribution that strongly favors short period systems $(\pi=-1)$. Similarly, the incidence of merger products (accounting for $8 \%$ in our standard simulation) varies by about a factor of two up or down $(4 \%-17 \%)$ when varying the slope of the initial period distribution.

Varying the mass ratio distribution from a distribution that strongly favors systems with unequal masses $(\kappa=-1)$ to a distribution that favors systems with equal masses $(\kappa=1)$ increases the fraction of binary products from $22 \%$ to $33 \%$. This results from the fact that binaries with comparable masses produce more massive binary products that are luminous enough to meet our brightness limit. The fraction of mergers varies only from $10 \%$ to $7 \%$. This is because of two effects that partially compensate each other. Systems with unequal masses are more likely to come in contact and merge. However, if systems with comparable masses merge they produce brighter objects. We refer to the discussion section in Paper I for further details.

The effect of changing the initial mass function is more modest. We find a variation of $27 \%-32 \%$ when adopting flatter $(\alpha=1.65)$ or a steeper ( $\alpha=3.05)$ steeper slope for the initial stellar mass function, respectively. When changing the adopted initial binary fraction down to $f_{\text {bin }}=0.3$ or up to $f_{\text {bin }}=1.0$ we find changes in the contamination by binary products between $15 \%$ and $37 \%$. When changing the adopted brightness limit from $10^{4}$ to $10^{5} L_{\odot}$ we find an increase in the incidence of binary products (to $34 \%$ ) and mergers (to $12 \%$ ).

Investigating the effects of the uncertain physical assumptions requires recomputing the grid of models. To speed up the computations, we recomputed them at lower resolution. In Table 1 we provide the results for variations of the physical assumptions as well as a reference simulation at the same resolution adopting our standard assumptions.

The dominant uncertainty affecting the predicted number of binary products is the accretion efficiency. This still remains one of the major uncertainties in binary evolutionary models. Attempts to constrain the efficiency using large samples of binary systems with accurately determined parameters remained inconclusive (e.g., de Mink et al. 2007 and references therein).

If we assume that all the mass lost by the primary star through Roche lobe overflow is accreted by the companion we find that the fraction of binary products increases to $34 \%$ because of the larger masses and thus luminosities of the binary products. Adopting highly non-conservative mass transfer by assuming that stars do not accrete after they have been spun up to their Keplerian rotation rate, which is typically after they have gained a few percent of their mass (Packet 1981), reduces the incidence of binary products to $18 \%$. Changing the adopted specific angular momentum of material that is lost from the system during interaction affects the number of mergers and whether the systems display detectable radial velocity variations after interaction. However, we find that this effect is minor compared to the other uncertain assumptions.

The number of mergers is mainly affected by the amount of mixing in the merger product. When the merger product is assumed to be fully mixed, the remaining lifetime is enhanced as a result of the fresh hydrogen that has become available in the central burning regions. The fraction of mergers is also sensitive to the adopted critical mass ratio for contact in systems where the donor star is on the main sequence. These two uncertainties are responsible for variations of $7 \%-12 \%$. We find that our predictions are neither very sensitive to the adopted amount of mass loss during the merger event nor to the adopted critical mass ratio for contact in systems where the donor star is a post main-sequence object.

\section{BINARY PRODUCTS IN OBSERVED SAMPLES}

The number of binary products present in observed stellar samples depends on the selection effects and the details of the underlying star-formation history. In populations younger than 2 Myr one might expect the overall contamination by binary products to be small, since only the most massive stars evolve fast enough to expand and interact. However, depending on the selection criteria, the contamination by binary products may be very significant. Even after only 1-2 Myr, the brightest star of a well populated star cluster is expected to be the product of binary evolution (Schneider et al. 2014). Therefore, in a sample consisting of the brightest stars of young star clusters, the contamination by mergers and other binary products may be significantly larger than predicted by our simulations for continuous star formation.

As discussed in Section 3.1, the inclusion or selection against known binaries can significantly change the relative number of binary products. Modeling this effect is very challenging since the selection against binaries is often not done in a systematic way. For example, in a typical sample, serendipitously discovered binaries described in the literature are excluded. Given the limited size of most samples these unsystematic selection effects cannot be ignored. Because of these sample-dependent difficulties, a quantitative comparison of our predictions with observations beyond the scope of this paper. However, we will make several general remarks.

An example of a large homogenous survey of massive stars is the VLT-FLAMES Tarantula Survey of massive stars (Evans et al. 2011). This ESO large program is a multi-epoch spectroscopic survey of 800 randomly selected early-type stars, among which $360 \mathrm{O}$ stars, in the Tarantula Nebula or 30 Doradus region of the Large Magellanic Cloud (Walborn 1984). The sample contains a mix of stellar populations with different ages including one that is at least 20 Myr old.

The fraction of $\mathrm{O}$ stars in this survey that are detected to be spectroscopic binaries is $0.35 \pm 0.03$ (Sana et al. 2013). Even though this multi-epoch spectroscopic survey was designed to systematically search for binaries, it is far from the idealized case demonstrated in Figure 3(b). After carefully modeling the specific biases for this survey, Sana et al. (2013) derive an intrinsic binary fraction of $0.51 \pm 0.04$. Based on our simulations we expect the contribution of post-interaction systems among the detected binaries to be very small. One may therefore tentatively compare this to the fraction of pre-interaction systems in our simulation for continuous star formation, e.g., Figure 1, where we find a remarkably similar fraction of $50 \%$.

The intrinsic binary fraction derived for the Tarantula Survey is lower than that derived for young galactic clusters, which is $0.7 \pm 0.1$ (Sana et al. 2012). The difference is small and might be purely the result of stochastic effects. However, our simulations show that this difference comes out naturally as a result of stellar evolution and binary interaction. The $\mathrm{O}$ stars in the young galactic survey have not had time to interact. Their binary fractions are expected to closely resemble conditions at birth, i.e., the onset of hydrogen burning. Instead, the Tarantula Survey contains early-type stars of a wide range of ages. The effects of stellar evolution and binary interactions have modified the intrinsic binary fraction.

A different large survey is the first VLT-FLAMES survey of massive stars (Evans et al. 2005), containing stars in our galaxy 
and the small and the large Magellanic cloud. This sample seems to be reasonably well characterized by continuous star formation. Brott et al. (2011b) used the early B-type stars in this survey to calibrate overshooting and the efficiency of rotationally induced mixing in stellar models for isolated single stars. Detailed modeling of the selection effects is required to estimate the contamination of binary products in this sample. However, based on our simulations one may expect the contaminated fraction to be in the order of almost a third of the sample. This is similar to the total fraction of stars with enhanced nitrogen abundances (box 1 and 3 in Figure 10 in Brott et al. 2011b, which account for $32.8 \%$ of the sample). This questions whether the observed nitrogen abundances are the result of rotational mixing processes operating in isolated rotating stars, or whether they are the result of binary interaction processes (Hunter et al. 2008).

Finally, a highly tentative comparison, which should be taken with great care, is the fraction of binary products or mergers with the observed fraction of peculiar stars. For example, the fraction of massive stars that are observed to be magnetic stars, i.e., about 10\% (Donati \& Landstreet 2009; Hubrig et al. 2011, 2013; Wade et al. 2011; Grunhut et al. 2012) is in the same order as the fraction of merger products that we expect. While this may be merely coincidence, several authors have speculated about the connection magnetic fields and binary interaction and mergers in particular (Ferrario et al. 2009).

\subsection{Characteristics of Binary Products}

Apart from statistical statements about the frequency of binary product, we can consider ways to identify individual binary products. There are several characteristics that give hints at a binary origin. Not all binary products are expected to show each of these characteristics and none of these characteristics uniquely signifies binary interaction.

1. The surface abundances of binary products may show signatures that indicate mixing or accretion of enriched gas. In particular, a depletion of fragile elements is expected, because these can only survive in the cooler outermost layers of the star. This concerns lithium, beryllium, boron, and fluorine (e.g., Langer et al. 2010). In more severe cases the burning products of hydrogen fusion can appear at the surface, i.e., an enhancement of helium, nitrogen, and sodium and a depletion of carbon and oxygen.

2. Binary products are likely to have peculiar rotation rates. In principle, rapid rotation is expected as a result of spin-up during mass accretion or a merger (Dufton et al. 2011; de Mink et al. 2013), of which Be/X-ray binaries are direct evidence (e.g., Rappaport \& van den Heuvel 1982). However, if binary products experience strong angular momentum loss, for example through magnetic braking, this may result in very slow rotators.

3. If binary interaction occurred recently there may be signs of shedded material in the circumstellar medium, either a (bipolar) ejection nebula (as in the case of the promising candidate for a merger product, the magnetic O6.5f?p star HD 148937, Leitherer \& Chavarria-K. 1987; Smith et al. 2004; Nazé et al. 2008, 2010) or a circum-binary disk (as seen for example around the interacting system RY Scuti, Grundstrom et al. 2007). The typical lifetime of such a nebula is expected to be around $10^{4} \mathrm{yr}$. Therefore one can expect several stars that have merged recently and thus still display a circumstellar remnant (Langer 2012).

4. It has been suggested that a merger process may lead to the generation of a magnetic field (e.g., Tout et al.
2008; Ferrario et al. 2009). Strong, large-scale fields can be detected through spectropolarimetry, as for example for the companion of the Plaskett star that has recently been spun up by mass accretion (e.g., Grunhut et al. 2013).

5. The supernova explosion of the primary star can break up the binary system, depending on the amount of mass lost from the system and the magnitude and orientation of the birth kick of the compact object (Blaauw 1961; Hoogerwerf et al. 2001). When a tight system is disrupted, the companion will acquire a high velocity. Such "runaway stars" can be detected by measuring either the radial velocity or the proper motion or by indirect evidence such as the presence of a bow shock or its remote location away from regions of star formation. In many cases the acquired velocities will be moderate, i.e., a few to tens of $\mathrm{km} \mathrm{s}^{-1}$ (e.g., Eldridge et al. 2011). These stars would not be classified as runaway stars; "walk-away star" may be a more appropriate term. However, they can travel tens to hundreds of parsecs from their birth location, because $1 \mathrm{~km} \mathrm{~s}^{-1} \approx 1 \mathrm{pc} \mathrm{Myr}^{-1}$ and these binary products may live for tens of Myr before they explode.

6. Binary products are not expected to have a nearby unaffected main-sequence companion.

7. If the former companion is still present it may be detectable in some cases through a UV excess (e.g., Gies et al. 1998; Peters et al. 2013) as in the case of a stripped helium star or through its $\mathrm{X}$-ray properties when the former companion is an accreting compact object.

8. Within coeval stellar populations such as star clusters, the binary products may stand out as the most luminous objects, possibly appearing younger than the age of the cluster. In this case they are the massive analogue of blue stragglers (van Bever \& Vanbeveren 1998; Chen \& Han 2009). For young star clusters, where the turn off is not well defined, binary products may still stand out as the upper mass tail of the stellar mass function (Schneider et al. 2014).

\section{CONCLUSION AND DISCUSSION}

Based on our simulations, we predict that a population of early-type stars characterized by continuous star formation is contaminated by stars that have experienced interaction with a companion. We estimate the fraction of binary products in such a sample to be $30_{-15}^{+10} \%$ and the fraction of mergers to be $8_{-4}^{+9} \%$. The error bars quoted here refer to the largest variation we obtained when varying the input distributions and the treatment of the physics of binary interaction with respect to our standard model. Even though larger variations cannot be excluded if multiple assumptions need to be adjusted in a way that systematically favors or disfavors binary products, we conclude-given our current understanding - that the contamination of a sample of early-type stars with binary products is considerable. This poses a potential challenge when using these samples for various applications.

In particular, our findings raise questions about the validity of tests and calibration of single stellar models against observational samples. We have shown that the commonly adopted strategy of excluding detected binaries from a sample is counterproductive. Removing systems with detectable radial velocity variations preferentially removes binary systems that have not yet interacted from the sample. Post-mass-transfer systems and mergers are left in the sample, accounting for $40_{-20}^{+25} \%$ in total; mergers in particular account for $15_{-10}^{+20} \%$. 
Our findings also shed new light on the interpretation of classes of peculiar stars by raising the question of whether binary interaction or mergers are responsible for the peculiarity. While objects such as binary products and stellar mergers in particular may sound exotic, we predict that they are quite common. This also raises concerns about our understanding of resolved and unresolved stellar populations, in particular the accuracy with which we can derive quantities such as the star formation rate, mass-to-light ratio, and initial mass function using population synthesis models which do not account for the effects of binarity.

Our results emphasize the need to further constrain the distribution of initial parameters, in particular the distribution of initial orbital periods. With regard to the physical assumptions, the certainty of our predictions is mainly limited by our poor understanding of the mass transfer efficiency and the treatment of contact systems and mergers. With regard to the design of observational surveys, our findings call for prioritizing efforts to devote great care to a careful and systematic inclusion or selection against known binary systems.

We acknowledge various members of the VLT-FLAMES massive stars consortium, PI: C. Evans for stimulating discussions. S.d.M. acknowledges support for this work by NASA through an Einstein Fellowship grant, PF3-140105 and a Hubble Fellowship grant HST-HF-51270.01-A awarded by STScI, operated by AURA, Inc., for NASA, under contract NAS 5- 26555.

\section{REFERENCES}

Antonini, F., Faber, J., Gualandris, A., \& Merritt, D. 2010, ApJ, 713, 90 Antonini, F., Lombardi, J. C., Jr., \& Merritt, D. 2011, ApJ, 731, 128 Blaauw, A. 1961, BAN, 15, 265

Braun, H., \& Langer, N. 1995, A\&A, 297, 483

Brott, I., de Mink, S. E., Cantiello, M., et al. 2011a, A\&A, 530, A115

Brott, I., Evans, C. J., Hunter, I., et al. 2011b, A\&A, 530, A116

Bruzual, G., \& Charlot, S. 2003, MNRAS, 344, 1000

Chen, X., \& Han, Z. 2009, MNRAS, 395, 1822

Chini, R., Hoffmeister, V. H., Nasseri, A., Stahl, O., \& Zinnecker, H. 2012, MNRAS, 424, 1925

Claeys, J. S. W., de Mink, S. E., Pols, O. R., Eldridge, J. J., \& Baes, M. 2011, A\&A, 528, A131

Conroy, C., Gunn, J. E., \& White, M. 2009, ApJ, 699, 486

de Mink, S. E., Langer, N., \& Izzard, R. G. 2011, BSRSL, 80, 543

de Mink, S. E., Langer, N., Izzard, R. G., Sana, H., \& de Koter, A. 2013, ApJ, 764, 166

de Mink, S. E., Pols, O. R., \& Hilditch, R. W. 2007, A\&A, 467, 1181

de Mink, S. E., Pols, O. R., Langer, N., \& Izzard, R. G. 2009, A\&A, 507, L1

Donati, J.-F., \& Landstreet, J. D. 2009, ARA\&A, 47, 333

Dray, L. M., \& Tout, C. A. 2007, MNRAS, 376, 61

Dufton, P. L., Dunstall, P. R., Evans, C. J., et al. 2011, ApJL, 743, L22

Ekström, S., Georgy, C., Eggenberger, P., et al. 2012, A\&A, 537, A146

Eldridge, J. J. 2012, MNRAS, 422, 794

Eldridge, J. J., Izzard, R. G., \& Tout, C. A. 2008, MNRAS, 384, 1109

Eldridge, J. J., Langer, N., \& Tout, C. A. 2011, MNRAS, 414, 3501

Evans, C. J., Smartt, S. J., Lee, J.-K., et al. 2005, A\&A, 437, 467

Evans, C. J., Taylor, W. D., Hénault-Brunet, V., et al. 2011, A\&A, 530, A108

Ferrario, L., Pringle, J. E., Tout, C. A., \& Wickramasinghe, D. T. 2009, MNRAS, 400, L71

Gaburov, E., Lombardi, J. C., \& Portegies Zwart, S. 2008, MNRAS, 383, L5

Gies, D. R., Bagnuolo, W. G., Jr., Ferrara, E. C., et al. 1998, ApJ, 493, 440

Glebbeek, E., Gaburov, E., de Mink, S. E., Pols, O. R., \& Portegies Zwart, S. F. 2009, A\&A, 497, 255

Glebbeek, E., Gaburov, E., Portegies Zwart, S., \& Pols, O. R. 2013, MNRAS, 434, 3497

Grundstrom, E. D., Gies, D. R., Hillwig, T. C., et al. 2007, ApJ, 667, 505

Grunhut, J. H., Wade, G. A., Leutenegger, M., et al. 2013, MNRAS, 428,1686
Grunhut, J. H., Wade, G. A., \& MiMeS Collaboration. 2012, in AIP Conf. Ser. 1429, Stellar Polarimetry: From Birth to Death, ed. J. L. Hoffman, J. Bjorkman, \& B. Whitney (Melville, NY: AIP), 67

Hamers, A. S., Pols, O. R., Claeys, J. S. W., \& Nelemans, G. 2013, MNRAS, 430, 2262

Hoogerwerf, R., de Bruijne, J. H. J., \& de Zeeuw, P. T. 2001, A\&A, 365, 49

Hubrig, S., Schöller, M., Ilyin, I., et al. 2013, A\&A, 551, A33

Hubrig, S., Schöller, M., Kharchenko, N. V., et al. 2011, A\&A, 528, A151

Hunter, I., Brott, I., Lennon, D. J., et al. 2008, ApJL, 676, L29

Hurley, J. R., Pols, O. R., \& Tout, C. A. 2000, MNRAS, 315, 543

Hurley, J. R., Tout, C. A., \& Pols, O. R. 2002, MNRAS, 329, 897

Izzard, R. G., Dray, L. M., Karakas, A. I., Lugaro, M., \& Tout, C. A. 2006, A\&A, 460,565

Izzard, R. G., Glebbeek, E., Stancliffe, R. J., \& Pols, O. R. 2009, A\&A, 508, 1359

Izzard, R. G., Tout, C. A., Karakas, A. I., \& Pols, O. R. 2004, MNRAS, 350,407

Kippenhahn, R., \& Meyer-Hofmeister, E. 1977, A\&A, 54, 539

Kozai, Y. 1962, AJ, 67, 591

Langer, N. 2012, ARA\&A, 50, 107

Langer, N., Brott, I., Cantiello, M., et al. 2010, in IAU Symp. 268, Light Elements in the Universe, ed. C. Charbonnel, M. Tosi, F. Primas, \& C. Chiappini (Cambridge: Cambridge Univ. Press), 411

Leitherer, C., \& Chavarria-K., C. 1987, A\&A, 175, 208

Leitherer, C., Ortiz Otálvaro, P. A., Bresolin, F., et al. 2010, ApJS, 189, 309

Li, Z., Zhang, L., \& Liu, J. 2012, MNRAS, 424, 874

Lu, P., Deng, L. C., \& Zhang, X. B. 2010, MNRAS, 409, 1013

Maeder, A., \& Meynet, G. 2000, A\&A, 361, 159

Mason, B. D., Hartkopf, W. I., Gies, D. R., Henry, T. J., \& Helsel, J. W. 2009, AJ, 137,3358

Mermilliod, J.-C. 1982, A\&A, 109, 37

Munari, U., Henden, A., Kiyota, S., et al. 2002, A\&A, 389, L51

Nazé, Y., Ud-Doula, A., Spano, M., et al. 2010, A\&A, 520, A59

Nazé, Y., Walborn, N. R., \& Martins, F. 2008, RMxAA, 44, 331

Nelson, C. A., \& Eggleton, P. P. 2001, ApJ, 552, 664

Nieuwenhuijzen, H., \& de Jager, C. 1990, A\&A, 231, 134

Packet, W. 1981, A\&A, 102, 17

Perets, H. B., \& Fabrycky, D. C. 2009, ApJ, 697, 1048

Peters, G. J., Pewett, T. D., Gies, D. R., Touhami, Y. N., \& Grundstrom, E. D. 2013, ApJ, 765, 2

Petrovic, J., Langer, N., \& van der Hucht, K. A. 2005, A\&A, 435, 1013

Podsiadlowski, P., Joss, P. C., \& Hsu, J. J. L. 1992, ApJ, 391, 246

Pols, O. R. 1994, A\&A, 290, 119

Pols, O. R., Schröder, K.-P., Hurley, J. R., Tout, C. A., \& Eggleton, P. P. 1998, MNRAS, 298, 525

Portegies Zwart, S. F., Baumgardt, H., Hut, P., Makino, J., \& McMillan, S. L. W. 2004, Natur, 428, 724

Potter, A. T., Chitre, S. M., \& Tout, C. A. 2012, MNRAS, 424, 2358

Rappaport, S., \& van den Heuvel, E. P. J. 1982, in IAU Symp. 98, Be Stars, ed. M. Jaschek \& H.-G. Groth (Dordrecht: Reidel), 327

Salpeter, E. E. 1955, ApJ, 121, 161

Sana, H., de Koter, A., de Mink, S. E., et al. 2013, A\&A, 550, A107

Sana, H., de Mink, S. E., de Koter, A., et al. 2012, Sci, 337, 444

Sana, H., \& Evans, C. J. 2011, in IAU Symp. 272, Active OB Stars: Structure, Evolution, Mass Loss, and Critical Limits, ed. C. Neiner, G. Wade, G. Meynet, \& G. Peters (Cambridge: Cambridge Univ. Press), 474

Schneider, F. R. N., Izzard, R. G., de Mink, S. E., et al. 2014, ApJ, 780, 117

Smith, N., Vink, J. S., \& de Koter, A. 2004, ApJ, 615, 475

Tout, C. A., Aarseth, S. J., Pols, O. R., \& Eggleton, P. P. 1997, MNRAS, 291,732

Tout, C. A., Wickramasinghe, D. T., Liebert, J., Ferrario, L., \& Pringle, J. E. 2008, MNRAS, 387, 897

Tylenda, R., Hajduk, M., Kamiński, T., et al. 2011, A\&A, 528, A114

Tylenda, R., \& Soker, N. 2006, A\&A, 451, 223

van Bever, J., \& Vanbeveren, D. 1998, A\&A, 334, 21

Vink, J. S., de Koter, A., \& Lamers, H. J. G. L. M. 2001, A\&A, 369, 574

Wade, G. A., Grunhut, J. H., Marcolino, W. L. F., et al. 2011, in IAU Symp. 272, Active OB Stars: Structure, Evolution, Mass Loss, and Critical Limits, ed. C. Neiner, G. Wade, G. Meynet, \& G. Peters (Cambridge: Cambridge Univ. Press), 220

Walborn, N. R. 1984, in IAU Symp. 108, Structure and Evolution of the Magellanic Clouds, ed. S. van den Bergh \& K. S. D. de Boer (Dordrecht: Reidel), 243

Wellstein, S., \& Langer, N. 1999, A\&A, 350, 148

Wellstein, S., Langer, N., \& Braun, H. 2001, A\&A, 369, 939

Zahn, J.-P. 1977, A\&A, 57, 383

Zhang, F., Li, L., Zhang, Y., Kang, X., \& Han, Z. 2012, MNRAS, 421, 743 